\title{
Enfoque histórico en la enseñanza del campo electro magnético
}

Historical approach in electromagnetic field teaching

\author{
Luis Manuel Tobaja*1,20, Julia Gil ${ }^{1}$ \\ ${ }^{1}$ Colegio Salesiano "María Auxiliadora", Badajoz, Spain \\ ${ }^{2}$ Universidad de Extremadura, Facultad de Ciencias, Badajoz, Spain
}

Recibida en 12 de Diciembre, 2017. Revisado en 23 de Abril, 2018. Aceptado en 25 de Abril, 2018.

\begin{abstract}
Actualmente se acepta que la introducción de la Historia de la Ciencia en la enseñanza de las ciencias ha resultado fructífera en la mejora de la actitud de los alumnos ante la ciencia y en la mejor comprensión de los métodos de la ciencia. Se ha realizado una experiencia didáctica con alumnos del último curso de bachillerato científico en la asignatura de física consistente en la enseñanza del campo electromagnético (CEM) e inducción magnética mediante una secuenciación histórica de los descubrimientos e interpretaciones teóricas ocurridos en la primera mitad del siglo XIX en este ámbito. Realizado un estudio cuasiexperimental con pretest y postest, se muestra que los alumnos que han seguido la secuenciación histórica mejoran más que el grupo de control en reconocimiento de la labor de los científicos, en comprensión de la metodología científica y en adquisición de conocimientos.
\end{abstract}

Palavras-chave: Historia de la Ciencia; Física; Electromagnetismo; Campo magnético.

Currently, it is widely accepted that the establishment of the History of Science in the education of general Sciences has been fruitful and productive developing into a better attitude on the students' side, as well as the improved understanding of the methods Science uses. Concerning this issue, a didactic and pedagogical training has been undertaken with a group of senior students in their last academic year. The experiment has been developed on the electromagnetic and the magnetic induction fields by means of a historical sequencing of discoveries likewise theoretical interpretations and judgements of the first part of the XIX century. Once the quasi experimental research with pre-test and postest was carried out, the results show that, in the case of those students who followed a historical sequencing, they have improved more than the control group in the areas of the recognition of Scientists' work, in the understanding of the Scientific Methodology and the knowledge acquisition.

Keywords: History of Science, Physics, Electromagnetism, Magnetic field.

\section{Introducción}

Según el informe del curso 2012/13 sobre el sistema universitario español del MECD, las carreras de la rama de ciencias en el decenio 2003-2004/2013-2014 han sufrido un descenso en el número de alumnos superior al $25 \%$ [1], en contraste con el aumento extraordinario de las carreras de la rama sanitaria, que casi han duplicado su número. No es un fenómeno novedoso, apareciendo recurrentemente desde hace más de dos décadas [2] a nivel mundial [3]. Esta significativa reducción de ingresados en las carreras científicas tiene su reflejo, y quizás su causa, en la percepción que los alumnos de secundaria tienen de las materias científicas. En la actualidad, el porcentaje de alumnos que eligen la rama de ciencias no alcanza el $45 \%$ del alumnado de bachillerato, siendo a comienzo de la década de los 90 superior al $60 \%$ (de los que casi dos tercios cursaban la opción científicotecnológica). Esta significativa inversión en el número de

*Correo electrónico: ltobaja@gmail.com alumnos de las modalidades científica y humanista en las últimas décadas contrasta con una realidad cotidiana cada vez más tecnificada, en la que los avances en todas las ramas de las ciencias crecen exponencialmente y se trasladan con celeridad a la sociedad. No es de extrañar que, tanto nacional como internacionalmente, se insista en mejorar la competencia científica no solo del alumnado, sino de toda la sociedad, para que puedan responder más acertada y responsablemente a los retos que surgen en la interrelación ciencia-sociedad.

La comunidad educativa no ha permanecido impasible ante estos hechos. En primera instancia se ha procurado dar explicación a un fenómeno evidentemente complejo y multicausal. En [4] se apela tanto a causas sociales como la estructura del sistema educativo, en la que las materias científicas tienen poca relevancia y carga horaria, tanto en primaria como en la secundaria obligatoria. Izquierdo Aymerich [5] coincide con el anterior y Guisasola, Gras-Mart et al. [6] en apuntar a la complejidad intrínseca de las ciencias, con un complejo cuerpo de 
conocimientos, ideas abstractas y procedimientos como motivo de desafecto por parte del alumnado. En todo caso, sin despreciar los motivos anteriores ni otras posibles causas, el discurso más fructífero ha tenido un carácter autocrítico, centrando la atención en las metodologías empleadas en la enseñanza de las ciencias tanto en la etapa universitaria como en la secundaria.

Desarrollando este último punto, desde el ámbito científico se han propuesto multitud de estrategias que pudiesen mejorar tanto el aprendizaje de las ciencias, como la visión y actitud que los alumnos tienen ante ésta, desde la introducción de prácticas de laboratorio a investigaciones guiadas o abiertas [7-8], pasando por la mejora de las habilidades de resolución de problemas [9] o el estudio de las ideas previas (misconceptions) del alumnado [10-14]. Una de las corrientes pedagógicas más importantes, en este sentido, ha sido el movimiento CTS (Ciencia, Tecnología y Sociedad), propulsor de la interrelación de los contenidos más tradicionales con otros relativos a aspectos sociales o tecnológicos. Dentro de este movimiento se puede incluir la propuesta de introducción de Historia de la Ciencia como apoyo, complemento o fundamento de los contenidos de las asignaturas científicas.

\section{Fundamentación y objetivos}

\subsection{Historia de la Ciencia}

La introducción de contenidos de Historia de la Ciencia es una opción inestimable para mostrar a los alumnos el modo en el que la sociedad se ha enfrentado a fenómenos fuera de su comprensión y ha encontrado explicaciones razonables y contrastables mediante el método científico. Plantear cuestiones que los científicos han tenido que responder y mostrar el camino seguido para ello, puede revelar el modo de trabajo de la comunidad científica: cómo se elaboran las teorías, cuál es el papel de los experimentos y las interpretaciones que se dan a estos; cómo estas interpretaciones se modifican en el tiempo haciendo cambios en las teorías que explican los fenómenos y el grado de certeza que puede tenerse, en un momento dado, de una determinada teoría [15]. Además pueden vislumbrarse las relaciones entre los diferentes miembros de la comunidad científica y entre estos y la sociedad, siendo estos lazos determinantes en el desarrollo de la ciencia, del mismo modo que esta es impulsora del desarrollo tecnológico y social.

Por el contrario, la descontextualización de los contenidos científicos, además de empobrecer al alumno, privándole de una perspectiva más amplia que le permita establecer relaciones más significativas entre los diferentes contenidos, intra e interdisciplinarmente, puede producir una pérdida de interés del alumnado por las ciencias [5].

El conjunto de conceptos, leyes y experiencias que actualmente componen los contenidos que se imparten sobre un tema cualquiera de ciencias es el resultado de un complejo proceso de elaboración de una explicación coherente de los fenómenos observados. En el camino para la elaboración de dicha explicación se han propuesto hipótesis que han resultado ser erróneas y otras que han debido matizarse y reescribirse con nuevos conocimientos aparecidos. En ocasiones nuevos formalismos matemáticos reformulan los conceptos originales actualizándolos o produciendo incluso cambios radicales en ellas [16]. Ese proceso forma parte inherente de los procedimientos de la ciencia y, en la práctica docente habitual, no se muestra. Esto revela a los alumnos una visión estática de la ciencia, en ocasiones dogmática, y puede ser una de las causas del desafecto de los alumnos hacia esta. Es comprensible no reconstruir el camino seguido para llegar a cada teoría actual en cada ocasión, muchas hipótesis ahora no serían comprensibles o crearían confusión en el alumnado, además de necesitar un tiempo que normalmente no se tiene en el desarrollo del currículo. Sin embargo, en ocasiones es conveniente y posible mostrar ese camino de aciertos y desaciertos realizado por las ciencias, más próximo a la realidad, en su evolución histórica [17].

Respecto a la componente afectiva del aprendizaje, conocer las dificultades, tanto personales como técnicas o sociales, que han tenido que enfrentar otras personas para hacer ciencia, puede mostrar el valor de la labor realizada; en ocasiones, luchando contra el corpus de ideas dominante, poniendo su vida o bienestar en riesgo [5], pudiéndose crear así un lazo empático con los alumnos actuales.

Por todo ello, varios autores [2], [18-20] consideran que la Historia de la Ciencia pueden contribuir a la adquisición de conocimientos, conceptuales y metodológicos, y mejorar la motivación del alumnado.

Este planteamiento de incorporación de la Historia de la Ciencia en el currículo de las materias de ciencias se ha traducido en diferentes propuestas de diverso calado y profundidad. En un primer nivel, muy superficial, los libros de texto en los niveles preuniversitarios han introducido información biográfica sobre los científicos más destacados, normalmente con notas al margen y añadidos al final de los temas denominados "Ciencia, Tecnología y Sociedad" [21]. Aunque es un primer paso, es dudoso que esto pueda ayudar al alumno a identificarse con los personajes mostrados y más aún que así puedan comprender mejor el método científico o la contribución de la ciencia al desarrollo social. Además, en los textos dedicados a los alumnos más jóvenes, que podrían ser más receptivos a estos contenidos interdisciplinares y "no totalmente" científicos, la información es muy parca y rara vez se encuentra contextualizada, en ocasiones mitificando a los científicos mostrados [22].

En otro nivel, se han introducido en los temarios de las asignaturas contenidos cuya finalidad primordial es mostrar la mutabilidad de las interpretaciones científicas de los hechos dentro de los parámetros del método científico. Por ejemplo, en la asignatura de física se incluyen la crisis de la física clásica, las diferentes interpretaciones de 
la luz o el cambio de paradigma del modelo geocéntrico al heliocéntrico.

Además, puede encontrarse en la bibliografía un alto número de experiencias novedosas realizadas en diferentes niveles educativos y disciplinas científicas en las que se han obtenido resultados positivos con la inclusión de la Historia de la Ciencia en el desarrollo curricular [17], [18], [23-26].

La enseñanza de las ciencias mediante la Historia de la Ciencia no es una empresa sencilla. Principalmente porque los actores que deben realizarla, los profesores, normalmente no tienen la suficiente formación para ello. No es fácil encontrar planes de estudio de las carreras universitarias de ciencias en las que exista alguna asignatura sobre este tema. Consecuencia de ello, es normal que los egresados en ciencias tengan un significativo déficit en este aspecto, dentro y fuera de nuestras fronteras [2]. Hay quienes alertan de un posible mal uso de la historia como interpretación de los hechos [2], [19], [27], riesgo que surge, por un lado al considerar los hechos sucedidos desde la perspectiva actual, no en el contexto en el que ocurrieron; y por otro al mostrar los hechos desde el punto de vista ejemplarizante o dogmático, desvirtuando en ambos casos la realidad al recalcar unas acciones o actitudes frente a otras.

\subsection{Objetivos y Presupuestos}

Considerándose el concepto de campo altamente abstracto, es lógico que los alumnos encuentren dificultades en su comprensión y uso; esto unido a las ideas previas que los alumnos tienen, por sus experiencias cotidianas, en el uso de las corrientes eléctricas o de los imanes [14], hacen que el CEM y la inducción sean unos temas de difícil estudio para el alumnado [28]. Por ello, ya se han realizado diferentes propuestas en la enseñanza y aprendizaje del CEM desde diferentes métodos didácticos [7], [29], algunos incluyendo la introducción de la Historia de la Ciencia [22], [30].

El objetivo de este artículo es presentar los resultados de una secuencia de enseñanza-aprendizaje del CEM e inducción magnética basada en el desarrollo histórico acontecido en dicho ámbito de la física en el siglo XIX para alumnos del último curso de bachillerato.

Se han planteado como objetivos la mejora en la comprensión de los procedimientos de las ciencias por los alumnos y en una actitud más positiva de estos ante la asignatura y ante las ciencias en general, sin que suponga una merma en el nivel de conocimientos que deben adquirirse.

Para la mejora de la comprensión del método científico se ha elegido el CEM y la inducción por no considerarse tradicionalmente un elemento generador de "crisis" científica. El currículo ya incorpora cuestiones de cambio de paradigma (modelo heliocéntrico, teorías sobre la luz...) y se ha considerado que es relevante para el alumno la formación de un paradigma nuevo, elaborado prácticamente a partir de unos pocos resultados experimentales. En este caso, la finalidad no es enseñar Historia de la Ciencia, sino, aprovechar esta para mostrar a los estudiantes la naturaleza de la ciencia [15].

Como aspectos fundamentales se han considerado las relaciones entre científicos y la no linealidad del desarrollo científico. En referencia al primer aspecto, se ha insistido en el intercambio epistolar entre científicos y la importancia de la publicación de resultados. Es conocida la correspondencia entre Ampére y Faraday y la colaboración entre Arago y Ampére o Gauss y Webber. Respecto a la publicación y difusión de resultados suele destacarse la impronta que las conferencias de Davy dejaron en el joven Faraday o el retraso de Henry en la publicación de sus resultados sobre inducción.

Insistir en la no linealidad del desarrollo científico es un aspecto tan crucial como espinoso, dado que, siendo una componente intrínseca del método científico, puede producir desorientación en los alumnos y sensación de zigzagueo [20], al romperse la unicidad entre experimento, explicación o interpretación de este y su formulación matemática.

Se han introducido apuntes biográficos de la mayoría de los científicos que participaron en el desarrollo de la teoría electromagnética clásica; evitando, en todo caso, tanto un estilo puramente anecdótico como heroico. También se han introducido como "actores" relevantes en la escena científica las academias e instituciones (Royal Institution, Royal Society, Academia de París), por su importante labor en la difusión y discusión del conocimiento científico.

Existen numerosos desarrollos de estudio del CEM, desde múltiples puntos de vista, algunos también incorporando una cierta perspectiva histórica, como las de Beléndez [30] o Furió y Guisasola [7]. Nuestra propuesta, que se puede encontrar desarrollada de modo escueto en el Anexo I, se fundamenta en el desarrollo cronológico de los acontecimientos poniendo un énfasis especial en las relaciones entre los científicos implicados y entre estos y las instituciones científicas de la época. Así, se insiste en la necesidad de interpretar y reescribir los conocimientos en virtud de nuevas teorías o experiencias realizadas a costa de una explicación más deductiva, fundamentada en las leyes generales de los fenómenos magnéticos.

\section{Diseño experimental}

Durante el curso 2014/2015 se ha realizado una experiencia didáctica con dos grupos de alumnos de $2^{\circ}$ de bachillerato. El de control, formado por 21 alumnos, recibió la instrucción del tema "Campo electromagnético e inducción" según la secuenciación tradicional y el grupo experimental, formado por 23 alumnos, siguió la secuenciación de dicho tema según una perspectiva histórica. En ambos grupos se ha empleado el mismo número de sesiones (15) y se han realizado las mismas actividades, modificando de un grupo a otro sólo el criterio con el que 
se ha organizado la secuencia didáctica y la información biográfica de los científicos que ha sido más intensa en el grupo experimental.

Con el fin de poner a prueba la hipótesis de trabajo de mejorar la comprensión de los procedimientos de las ciencias por los alumnos y alcanzar una actitud más positiva de estos ante la materia y ante las ciencias en general, se utilizó un diseño cuasi-experimental con medidas de pretest y postest midiendo dos aspectos, el actitudinal y el conceptual.

En el pretest de valoración de actitudes se pedía a los alumnos la ordenación, según interés, de las asignaturas cursadas y de una serie de doce profesiones. También debían relacionar el nombre de unos científicos con alguno de sus descubrimientos. Por último debían valorar de "totalmente en desacuerdo" a "totalmente de acuerdo" una serie de afirmaciones relacionadas con la ciencia y sus usos.

En el pretest de conocimientos tenían que responder a un test de respuesta múltiple con diez preguntas sobre contenidos básicos de imanes y corrientes. En ambos grupos el pretest se realizó un mes antes de comenzar la unidad sobre campo magnético.

El postest de actitudes volvía a pedir relacionar el nombre del científico con su descubrimiento, en este caso sólo para los tratados en el tema; identificar las fotografías de cuatro de los científicos mencionados en el tema (ambos grupos disponían en sus apuntes de todas las fotos mostradas en el postest) y una serie de preguntas de valoración del tema visto y de afirmaciones relacionadas con la ciencia y sus usos, en parte ya preguntadas anteriormente en el pretest.

El postest de conocimientos constaba de una primera parte con tres respuestas de verdadero o falso, cuatro preguntas de respuesta múltiple y dos problemas numéricos. En ambos grupos el postest se realizó diez días después de haber concluido el tema.

\section{Resultados}

\subsection{Pretest de actitudes}

En la Gráfica 1 se presentan los resultados obtenidos al solicitar a los alumnos que relacionen una serie de científicos conocidos con alguno de sus descubrimientos o invenciones. El número medio de aciertos es de 2,65/10 y solo tres alumnos aciertan más de la mitad. Sólo se supera el $70 \%$ de aciertos cuando los ítems están relacionados con Einstein y M. Curie. El científico menos reconocido es Ampère y su teoría del electromagnetismo. Le siguen Faraday y Tesla, siendo Oersted el científico con mayor porcentaje de respuestas en blanco. Puede resultar llamativo el bajo porcentaje de acierto sobre Newton por su trabajo en la dispersión de la luz blanca. Cabe destacar, que excepto en los ítems relacionados con M. Curie y Faraday, el índice de acierto en el grupo experimental ha sido menor que en el grupo de control.
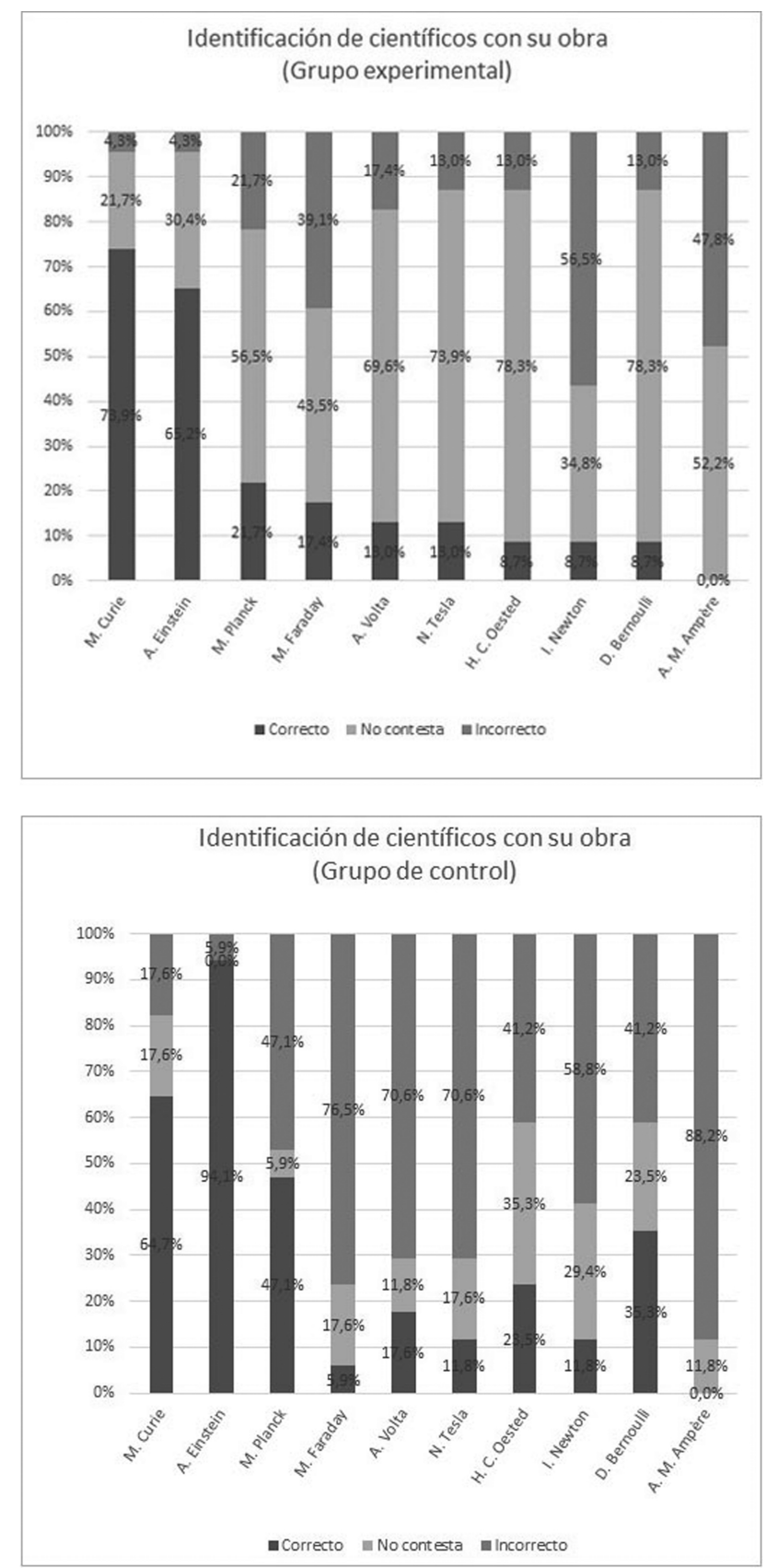

Gráfica 1: Identificación, por parte de los alumnos, de los científicos con su obra.

Por otro lado, respecto a las afirmaciones sobre la ciencia, más del $80 \%$ está de acuerdo o totalmente de acuerdo con las frases "Todo el mundo debería tener un mínimo de conocimientos sobre ciencia", "La investigación científica es útil a la sociedad" y "El desarrollo de un país está relacionado con su nivel en investigación científica". Más controvertida es la respuesta a "La ciencia origina problemas sociales y morales", donde la respuesta mayoritaria $(35 \%)$ se encuentra en el "Ni de acuerdo ni en desacuerdo".

El $97.5 \%$ de los alumnos discrepa con la frase "la ciencia es cosa de hombres, no de mujeres" y en la misma 
proporción muestran su desacuerdo con "los científicos son frikis". Diferente respuesta obtiene la cuestión "Para ser científico hay que ser más inteligente que la media" en la que se muestran en acuerdo un $35.9 \%$ y en desacuerdo un $28.2 \%$.

$\mathrm{Al}$ respecto de la ciencia como profesión, las respuestas están en consonancia con la VI encuesta de percepción social de la ciencia de 2012 del Fecyt [31], como puede apreciarse en la Tabla 1. Los datos dan a entender que los alumnos consideran la ciencia como valiosa y necesaria para la sociedad, pero que no está suficientemente remunerada (¿para el esfuerzo que supone dedicarse a ella?), ni tiene suficiente consideración social. En la misma línea creen que no es un modo sencillo de encontrar trabajo, aspecto que no concuerdan con los datos publicados por la RSEF [32] y que, evidentemente, desincentiva dedicarse a las ciencias como profesión. Esto contrasta con la valoración realizada de diferentes profesiones, donde, el $55 \%$ de los alumnos elegían la profesión de científico en primer, segundo o tercer lugar, por delante de médico con un $52.5 \%$ (si bien es elegida más veces en primer lugar, por un $27 \%$ de los alumnos), e ingeniero con un $40 \%$. Cabe resaltar que en este bloque de cuestiones, la opinión de los alumnos del grupo experimental fue más negativa que la del grupo de control.

Analizados los resultados anteriores mediante la prueba U de Mann-Whitney para la igualdad de medias en muestras independientes, no se han encontrado diferencias significativas entre las opiniones del grupo de control y el experimental en ninguno de los aspectos referidos.

\subsection{Pretest de conocimientos}

El análisis de los resultados del pretest de conocimientos indica que no existen diferencias significativas entre los grupos experimental y de control sobre los contenidos del tema de campo magnético e inducción, habiéndose obtenido como valores medios, 4,53/10 y 4,19/10, respectivamente. En la Gráfica 2 aparecen representadas las distribuciones de los datos obtenidos en el pretest para ambos grupos, donde se puede observar que partimos de grupos homogéneos.

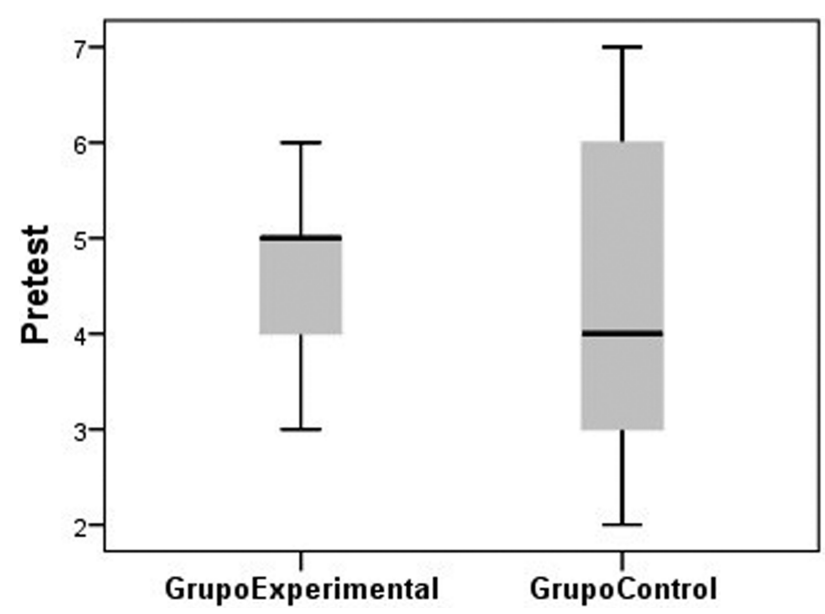

Gráfica 2: Resultados pretest de conocimientos

Destacar que de las respuestas de los alumnos se deduce que estos desconocían cualidades básicas de los imanes como los materiales con los que interaccionan o el tipo de interacción, así como el concepto de líneas de campo. En general, manifestaban no haber realizado experiencias de laboratorio en dicho campo.

\subsection{Postest de actitudes}

La primera cuestión del postest de actitudes solicita a los alumnos la identificación de los descubrimientos realizados con sus respectivos científicos, en este caso, relativos exclusivamente al electromagnetismo (ver Gráfica 3). La media de acierto ha sido superior en el grupo experimental que en el de control, $2.85 / 5$ frente a $2.1 / 5$.

La segunda pregunta del postest de actitudes consistía en reconocer a cuatro científicos a través de una imagen de su rostro. Combinada con las respuestas de la primera pregunta nos puede dar una idea de conjunto de la afinidad afectiva con los científicos aparecidos durante el tema. El grupo experimental identifica mejor que el de control los rostros de los científicos estudiados en tres de las cuatro imágenes mostradas (Ampère, Oersted y

Tabla 1: Valoración actitudes pretest sobre la ciencia y comparación resultados Fecyt 2012

\begin{tabular}{|c|c|c|c|c|c|c|c|c|c|}
\hline \multicolumn{4}{|c|}{ Encuesta Fecyt } & \multicolumn{6}{|c|}{ Encuesta de elaboración propia } \\
\hline $\begin{array}{l}\text { Ser investiga- } \\
\text { dor es una pro- } \\
\text { fesión... }\end{array}$ & $\begin{array}{l}0 \\
\frac{0}{2} \\
\frac{D}{Z}\end{array}$ & $\begin{array}{l}0 \\
\dot{0} \\
0\end{array}$ & $\stackrel{0}{\stackrel{0}{0}}$ & 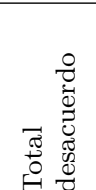 & 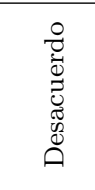 & 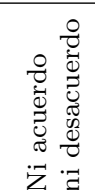 & 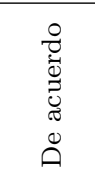 & 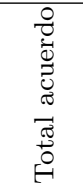 & \\
\hline Atractiva & $7.8 \%$ & $33.1 \%$ & $59.0 \%$ & $2.5 \%$ & $17.5 \%$ & $32.5 \%$ & $27.5 \%$ & $20 \%$ & Quiero ser científico \\
\hline $\begin{array}{l}\text { Remuneración } \\
\text { económica }\end{array}$ & $22.9 \%$ & $49.3 \%$ & $27.8 \%$ & $10.3 \%$ & $25.6 \%$ & $38.5 \%$ & $23.1 \%$ & $2.6 \%$ & $\begin{array}{l}\text { Dedicarse a la ciencia está bien } \\
\text { pagado }\end{array}$ \\
\hline $\begin{array}{l}\text { Reconocimien- } \\
\text { to social }\end{array}$ & $7.8 \%$ & $54.6 \%$ & $37.5 \%$ & $13.1 \%$ & $28.9 \%$ & $42.1 \%$ & $15.8 \%$ & $0 \%$ & $\begin{array}{l}\text { Dedicarse a la ciencia es un mo- } \\
\text { do de tener reconocimiento so- } \\
\text { cial }\end{array}$ \\
\hline & & & & $10.0 \%$ & $30.0 \%$ & $35.0 \%$ & $22.5 \%$ & $2.5 \%$ & $\begin{array}{l}\text { Dedicarse a la ciencia es un mo- } \\
\text { do fácil de encontrar trabajo }\end{array}$ \\
\hline
\end{tabular}


Identificación de científicos con su descubrimiento

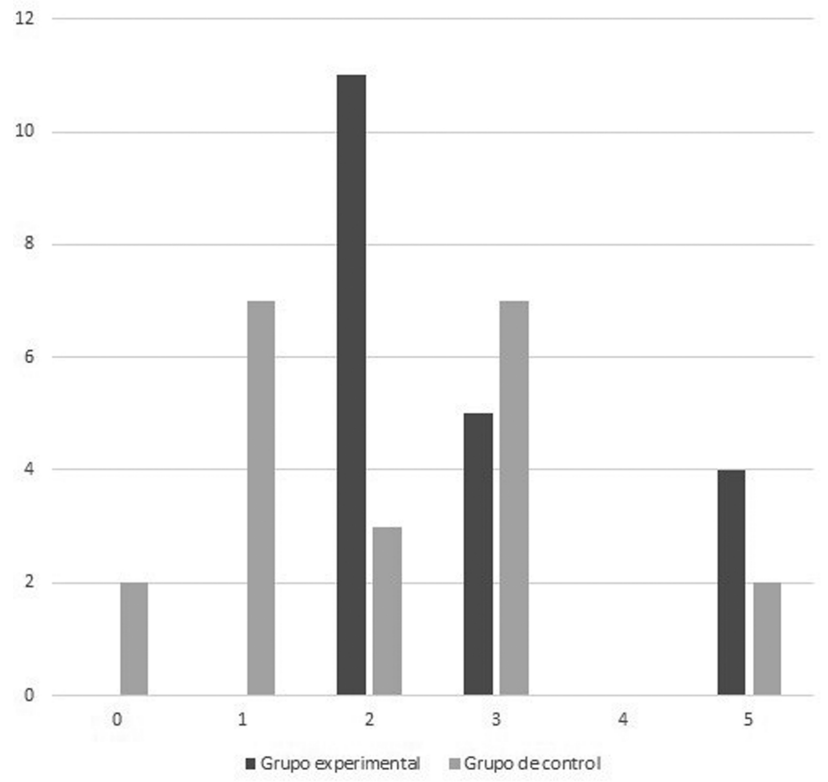

Gráfica 3: Acierto en la identificación de cada científico con su descubrimiento en el postest de actitudes

Maxwell). La media de aciertos en el grupo experimental fue de 1.83/4 frente al 1.10/4 del grupo de control.

En la última cuestión del postest de actitudes se solicitaba a los alumnos una valoración global del tema. Los resultados muestran que no existen diferencias estadísticamente significativas en la valoración (3.8/5) de ambos grupos. Esto puede considerarse un buen dato, ya que el grupo experimental tuvo una mayor carga de contenidos que el grupo de control, al añadirse a los contenidos conceptuales propios del tema los datos históricos de los científicos implicados. Además, se les exigió un esfuerzo conceptual de reelaboración de los contenidos que no realizó el grupo de control.

Con el fin de valorar el impacto de la propuesta aquí presentada se ha calculado la ganancia normalizada de las respuestas a una serie de preguntas repetidas en el pretest y el postest. Según Hake [33], se define la ganancia normalizada $\mathrm{G}$ como la diferencia entre las puntuaciones postest y pretest entre el incremento máximo de puntuación:

$$
G=\frac{\overline{\text { postest }}-\overline{\text { pretest }}}{\overline{\text { puntuación máxima }}-\overline{\text { pretest }}}
$$

Este índice ha sido empleado para valorar el grado de aprendizaje conceptual alcanzado por los alumnos en otros estudios. La Tabla 2 muestra las ganancias en ambos grupos para una serie de preguntas actitudinales.

La cuestión "Quiero ser científico" obtiene en ambos grupos una ganancia negativa, aunque en mayor medida en el grupo de control. Suponemos que la causa puede encontrarse en factores externos a este estudio, basados quizás, en la definición que los alumnos van encontrando de sus salidas profesionales a lo largo que avanza el curso.
Tabla 2: Ganancia de las respuestas, de los grupos experimental y de control, en preguntas de actitud hacia la ciencia

\begin{tabular}{lll}
\hline Pregunta & \multicolumn{1}{c}{$\begin{array}{c}\text { Grupo } \\
\text { Experimental }\end{array}$} & $\begin{array}{c}\text { Grupo de } \\
\text { Control }\end{array}$ \\
\hline Quiero ser científico & $-0,13$ & $-0,21$ \\
\hline Me gusta la física & 0,00 & $-0,02$ \\
\hline $\begin{array}{l}\text { Estudiar física me produce ansie- } \\
\text { dad }\end{array}$ & $-0,30$ & $-0,11$ \\
\hline La ciencia no es cosa de mujeres & $-0,04$ & $-0,05$ \\
\hline Los científicos son "frikis" & 0,28 & 0,15 \\
\hline $\begin{array}{l}\text { Para ser científico hay que ser } \\
\text { más inteligente que la media }\end{array}$ & $-0,04$ & $-0,11$ \\
\hline $\begin{array}{l}\text { La investigación científica es útil } \\
\text { a la sociedad }\end{array}$ & 0,52 & $-0,74$ \\
\hline $\begin{array}{l}\text { La ciencia origina problemas so- } \\
\text { ciales y morales }\end{array}$ & $-0,13$ & $-0,18$ \\
\hline $\begin{array}{l}\text { El desarrollo de un país está re- } \\
\text { lacionado con su nivel en investi- } \\
\text { gación científica }\end{array}$ & $-0,30$ & $-0,22$ \\
\hline
\end{tabular}

El estudio realizado parece no haber conseguido mejorar el gusto por la física de los alumnos. Ambos grupos mantienen, prácticamente, las puntuaciones del pretest en el postest. Sin embargo, ambos grupos disminuyen su percepción de ansiedad ante la asignatura, en mayor medida en el grupo experimental, resultado que se considera positivo y dentro de los objetivos del estudio realizado.

Las respuestas a las tres últimas preguntas de la Tabla 2 muestran una mejora de la visión de los alumnos del grupo experimental sobre la utilidad y bondad del desarrollo científico para la sociedad. Por otra parte, la ganancia negativa en la última pregunta puede interpretarse considerando que el tema ha mostrado una visión más globalizada de la ciencia, no incidiendo exclusivamente cada descubrimiento en un país en concreto.

Aunque los científicos mostrados en el tema eran todos hombres, la opinión sobre la cuestión "La ciencia no es cosa de mujeres" no se ha modificado respecto al pretest. Esta cuestión parece ajena a nuestro estudio, imputando la igualdad de opiniones en ambos test a causas educativas o sociales a favor de la igualdad de hombres y mujeres.

Uno de los resultados destacables aparece en la cuestión "Los científicos son "frikis"", donde el grupo experimental obtiene una ganancia de 0'28 frente a la del grupo de control de 0'15. No consideramos el término coloquial "friki" con connotación negativa, aunque sí refleja una visión del científico como una persona inusual, que no se comporta o vive de modo convencional. Puede interpretarse que los alumnos, al conocer más de cerca la vida de alguno de ellos, dejan de considerarlos "normales". También es posible que refleje la idea aceptada de una persona "friki" como alguien con dedicación desmesurada a una determinada afición, en este caso la ciencia.

Otro de los bloques de preguntas del postest indagaba en el interés de los alumnos por las biografías de los científicos en las que el grupo experimental ha obtenido mayores medias que el grupo de control.

El último de los bloques de preguntas del postest cuestionaba a los alumnos sobre los métodos de la ciencia. 
En la Tabla 3 pueden verse las puntuaciones medias obtenidas por ambos grupos.

Puede apreciarse cómo, en todos los casos, las respuestas dadas por los alumnos del grupo experimental están más de acuerdo con los principios del método científico que las del grupo de control. Así mismo, consideran la ciencia como una labor colectiva y los descubrimientos científicos fruto del trabajo y dedicación de los científicos y suponen que las leyes científicas pueden modificarse en el transcurso del tiempo, siempre en mayor medida que el grupo de control.

\subsection{Postest de conocimientos}

En la Gráfica 4 se presenta, en diagrama de caja, la distribución de los valores postest para ambos grupos, que una vez analizados con la prueba U de Mann-Whitney no nos permite rechazar la hipótesis nula de igualdad de medias y podemos aceptar que no hay diferencia estadísticamente significativa entre ambas distribuciones.

Los resultados obtenidos en la prueba postest de conocimientos arrojan una media para el grupo experimental de 5.79 y para el grupo de control de 5.00. No debemos olvidar que partíamos de resultados superiores para el grupo experimental en el pretest de conocimientos, pero hay que resaltar que este grupo ha aumentado la diferencia, como así lo indica la ganancia normalizada calculada para ambos grupos, $\mathrm{G}=0.20$ para el grupo experimental y $\mathrm{G}=0,09$ para el grupo de control (ver Gráfica 5).

\section{Conclusiones}

Los resultados obtenidos indican que ha habido un mayor aprendizaje de los contenidos ya que la ganancia normalizada de aprendizaje sobre los contenidos académicos del tema tratado es $\mathrm{G}=0.20$ para el grupo experimental

Tabla 3: puntuaciones medias de los alumnos en el postest de actitudes

\begin{tabular}{lll}
\hline Pregunta & $\begin{array}{c}\text { Grupo } \\
\text { Experimental }\end{array}$ & $\begin{array}{c}\text { Grupo de } \\
\text { Control }\end{array}$ \\
\hline $\begin{array}{l}\text { El objetivo del trabajo científico } \\
\text { es el establecimiento de relaciones } \\
\text { matemáticas (leyes) entre magni- }\end{array}$ & 3,10 & 3,24 \\
tudes físicas & \\
\hline $\begin{array}{l}\text { A menudo los descubrimientos } \\
\text { científicos se han producido por }\end{array}$ & 2,85 & 3,52 \\
casualidad o azar & \\
\hline $\begin{array}{l}\text { Los descubrimientos científicos } \\
\text { son fundamentalmente obra del } \\
\text { trabajo personal de científicos } \\
\text { concretos }\end{array}$ & \\
\hline $\begin{array}{l}\text { El trabajo de un científico nor- } \\
\text { malmente no es influido por el de } \\
\text { otros científicos }\end{array}$ & \\
\hline $\begin{array}{l}\text { Los conceptos y modelos científi- } \\
\text { cos son fieles reflejos de la reali- }\end{array}$ & 3,55 & \\
dad & & \\
\hline $\begin{array}{l}\text { Una vez que una ley científica se } \\
\text { establece no se modifica más }\end{array}$ & 1,50 & \\
\hline
\end{tabular}

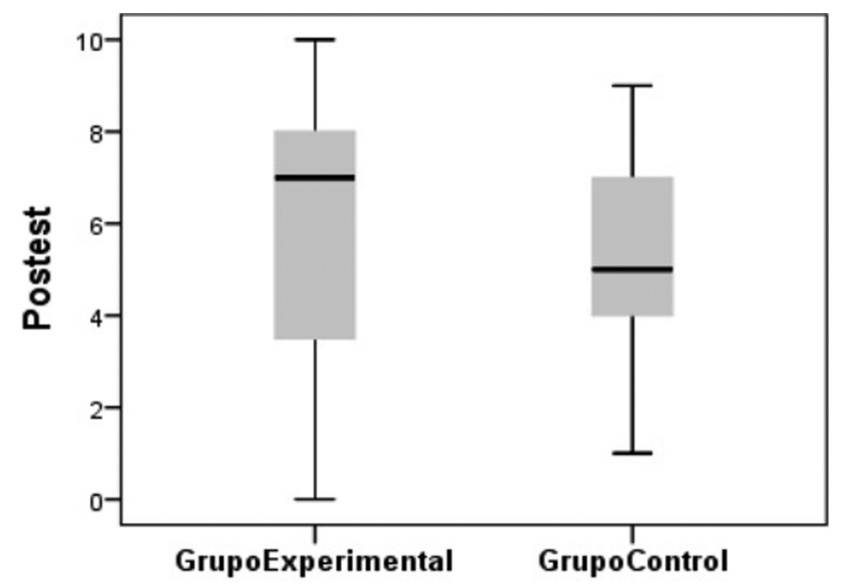

Gráfica 4: Valores postest de conocimientos

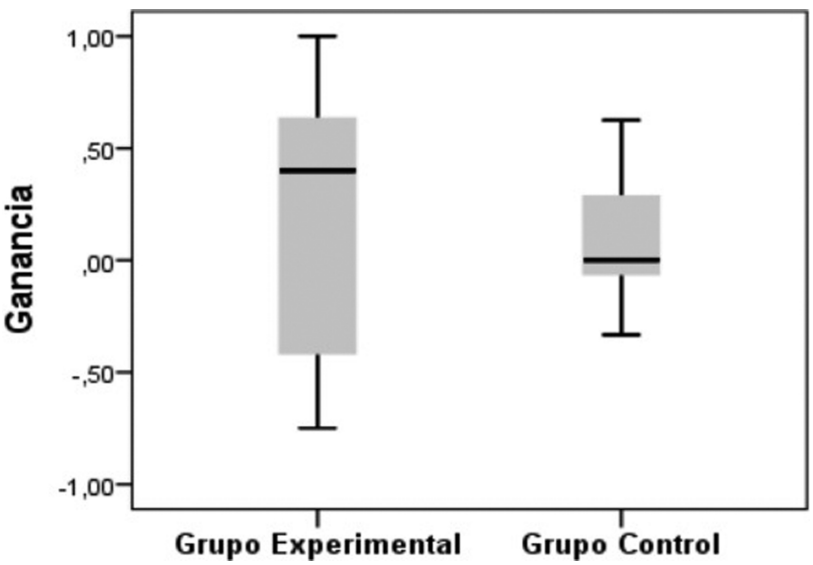

Gráfica 5: Ganancia normalizada del postest de conocimientos

frente $\mathrm{G}=0,09$ para el grupo de control, sin olvidar que el tiempo utilizado en ambas secuencias de aprendizaje ha sido el mismo. Además la valoración del alumnado del tema impartido siguiendo la secuencia histórica ha sido igual a la del grupo de control. Estos dos resultados se perciben como positivos debido a la mayor densidad conceptual del desarrollo histórico, al que se le añaden los contenidos propiamente históricos a los puramente conceptuales, además de suponer un mayor esfuerzo de elaboración por parte del alumnado.

En el aspecto actitudinal ante la ciencia, se obtienen mejores resultados en el grupo experimental que en el de control. Reconocen en mayor medida a los científicos implicados en el descubrimiento de las leyes que rigen el CEM, y asocian mejor a estos con el descubrimiento realizado por cada uno. Además reconocen más interés por sus biografías y los tienen en mejor consideración. También ofrecen mejores resultados en actitudes hacia la ciencia, reduciendo el valor de la suerte en los descubrimientos científicos y considerando en mayor medida la labor colaborativa de la ciencia y la mutua influencia entre unos científicos y otros.

Con los datos obtenidos en el postest de actitudes podemos afirmar, considerando la incertidumbre causada 
por la baja muestra estadística, que el objetivo de mejorar la actitud de los alumnos ante la ciencia y los científicos se puede alcanzar.

En otro orden de cosas, quizás fuese interesante resolver el déficit en historia y epistemología de las ciencias en los futuros científicos egresados de la universidad; también, por supuesto, para los futuros maestros de primaria que impartan Ciencias Naturales [34]. Aunque faltaría resolver aún la carencia de los docentes actualmente en activo que no han recibido la suficiente formación en este campo de la ciencia.

\section{Material suplementario}

El siguiente material suplementario está disponible en línea:

Anexo 1

\section{Referencias}

[1] Datos básicos Datos Sistema Universitario español. Curso 2013-2014. (Secretaría General Técnica. Subdirección General de Documentación y Publicaciones. Ministerio de Educación, Cultura y Deporte, 2014).

[2] M. R. Matthews, Enseñanza de Las Ciencias 12, 255 (1994).

[3] D. Gil Pérez, B. Macedo, J. Martínez-Torregrosa, C. Sifredo, P. Valdés y A. Vilches, ¿Cómo promover el interés por la cultura científica? (Oficina Regional de Educación de la UNESCO para América Latina y el Caribe OREALC/UNESCO, 2005).

[4] M. F. Petit Pérez y J. Solbes Matarredona, Enseñanza de las Ciencias 30, 55 (2012).

[5] M. Izquierdo Aymerich, Aula de Innovación Educativa 27, 37 (1994)

[6] J. Guisasola, A. Gras-Mart, J. Martínez-Torregrosa, J. M. Almudí y C. Becerra-Labra, Revista Brasileira de Ensino de Física 26, 197 (2004).

[7] C. Furió y J. Guisasola, Enseñanza de las Ciencias 19, 199 (2001).

[8] M. Picquart, Latin American Journal of Physics Education 2, 29 (2008).

[9] C. Becerra-Labra, A. Gras-Mart y J. MartínezTorregrosa, Revista Brasileira de Ensino de Física 32, e2401 (2010).

[10] J. Carrascosa Alís, y D. Gil Pérez, Enseñanza de las Ciencias 10, 314 (1992).

[11] F. G. Criscuolo, Enseñanza de las Ciencias 5, 231 (1987).

[12] J. Clement, D. E. Brown y A. Zietsman, Int. J. Sci. Educ. 11, 554 (1989).

[13] M. Montanero, M. I. Suero, A. L. Pérez y P. J. Pardo, Physics Education 37, 318 (2002).

[14] A. M. Pérez, Ingeniería y Desarrollo 6, 5 (1999).

[15] A. Caamaño, Alambique 8, 43 (1996).

[16] M.I. Aymerich, C. Merino, M.Q. Gatica y J.V. Segura, Alambique 48, 78 (2006).

[17] J. Solbes y M.J.T. Ribes, Enseñanza de las Ciencias 19, 151 (2001).

[18] M.M.Á. Lires, A.A. Correa, U.P. Rodríguez y J.F.S. Marzoa, Enseñanza de las Ciencias 31, 213 (2013).
[19] R. Gagliardi, Enseñanza de Las Ciencias 6, 291 (2006).

[20] R.D. Castillo y M.G. Díaz, Revista Iberoamericana de Educación 38, 1 (2006).

[21] J. Velasco, Revista de Investigación 64, 63 (2008).

[22] V.S. Dias y R. De A. Martins, Ciência \& Educação (Bauru) 10, 517 (2004).

[23] C. De Hosson, Enseñanza de Las Ciencias, 29, 115 (2011).

[24] D. P. Rodríguez Pineda y J. González Florez, Tecné, Episteme y Didaxis, 121 (2002).

[25] L.P. De Eulate, Revista Alambique 8, 71 (1996).

[26] R. Gagliardi y A. Giordan, Enseñanza de las Ciencias 4, 253 (1986).

[27] C.A. Jaime, A.F. Cachapuz, J.F. Praia, D.G. Pérez y I. Fernández, Enseñanza de Las Ciencias 20, 477 (2002).

[28] D. E. Gallego Quiceno, in $5^{\circ}$ Congreso Nacional de Enseñanza de la Física (Bogotá, 2011). p. 79.

[29] J. Guisasola, J.M. Almudi, M. Ceberio y J.L. Zubimendi, International Journal of Science and Mathematics Education 7, 699 (2009).

[30] A. Beléndez, Revista Brasileira de Ensino de Física 30 , e2601 (2008).

[31] FECYT, VI Encuesta de Percepción Social de la Ciencia 2012 (FECYT, Madrid, 2012).

[32] T.L. Martínez, Revista Española de Física 29, 41 (2015).

[33] R. R. Hake, Am. J. Phys. 66, 1 (1998).

[34] M.I. Aymerich, A.E. Profitós y A. Adúriz-Bravo, Enseñanza de las Ciencias 20, 465 (2002). 